\title{
ResearchOnline@JCU
}

This is the Accepted Version of a paper published in the Journal: Biomedicine and Pharmacotherapy

Haleagrahara, Nagaraja, Miranda-Hernandez, Socorro, Alim, Md Abdul, Hayes,

Linda, Bird, Guy, and Ketheesan, Natkunam (2017) Therapeutic effect of quercetin in collagen-induced arthritis. Biomedicine \& Pharmacotherapy, 90. pp. 38-46.

http://dx.doi.org/10.1016/j.biopha.2017.03.026

(C) 2015. This manuscript version is made available under the CC-BY-NC-ND 4.0 license http://creativecommons.org/licenses/by-nc-nd/4.0/

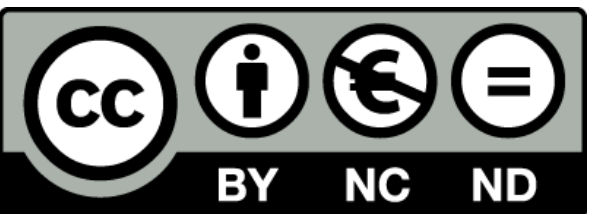




\section{Therapeutic effect of quercetin in collagen-induced arthritis}

Nagaraja Haleagrahara a,b,*, Socorro Miranda-Hernandez ${ }^{a}$, Md Abdul Alima,b, Linda Hayes $^{\mathrm{a}}$, Guy Bird ${ }^{\mathrm{c}}$, Natkunam Ketheesan ${ }^{\mathrm{a}, \mathrm{b}}$

${ }^{a}$ College of Public Health, Medical and Veterinary Sciences, James Cook University, James Cook Drive, Townsville, Queensland 4811, Australia

${ }^{\mathrm{b}}$ Australian Institute of Tropical Health and Medicine, James Cook University, James Cook Drive, Townsville, Queensland 4811, Australia

'JCU Veterinary Hospital, James Cook University, Townsville Queensland 4811, Australia

${ }^{*}$ Corresponding author

Dr Nagaraja Haleagrahara

College of Public Health, Medical and Veterinary Sciences,

James Cook University,

James Cook Drive, Townsville,

Queensland 4811, Australia

Tel: +61747816370

Email: haleagrahara.nagaraja@jcu.edu.au 


\begin{abstract}
Quercetin, a bioactive flavonoid with anti-inflammatory, immunosuppressive and protective properties, is a possible agent for Rheumatoid Arthritis (RA). Collageninduced arthritis (CIA) is the most common animal model to study the pathogenesis of RA. This study analysed the therapeutic role of quercetin in collagen-induced arthritis in C57BL/6 mice. Animals were allocated into five groups: negative control, positive control (arthritis), arthritis+methotrexate, arthritis+quercetin and arthritis + methotrexate+quercetin. Weight, oedema, joint damage and cytokines production were assessed to determine the therapeutic effect of quercetin. This study demonstrates for the first time that quercetin has anti-inflammatory and protective roles in vivo in CIA. We also report that quercetin and methotrexate administered concurrently show no more protection than a single agent. Quercetin as a monotherapeutic agent showed the lowest degree of joint inflammation and the highest protection. Less severe disease in animals treated with quercetin was associated with decreased levels of TNF- $\alpha$, IL-1 $\beta$, IL-17 and MCP-1. In conclusion, this study supports the argument that quercetin produces better results with no known toxicity than methotrexate in safeguarding joints from arthritic inflammation in mice. Quercetin could be an alternative treatment for RA because it modulates the main pathogenic pathways of RA.
\end{abstract}

Keywords:

Collagen induced arthritis, Rheumatoid arthritis, Inflammation, Quercetin, Cytokines, C57BL/6 


\section{Introduction}

Rheumatoid arthritis (RA) is a chronic inflammatory and degenerative joint disease affecting all ages, sexes and races [1,2]. RA is characterized by swelling, pain and stiffness in joints but it can also damage the heart, eyes, lungs, kidneys, and skin [3-7]. Patients with RA suffer progressive disability and increased risk of death [8]. Genetic, hormonal and environmental factors have been related to RA [9-12].

Advances to develop new treatments for RA have relied on animal models. The collagen-induced arthritis (CIA), model of RA, closely replicates the pathogenesis of the disease. Currently, therapeutic products for RA include: 1) Conventional synthetic disease-modifying antirheumatic drugs (DMARDs) such as methotrexate (Mtx), sulfasalazine, leflunomide and hydroxychloroquine; 2) Biological DMARDs such as TNF inhibitors, anti-B cell, anti-T cell and anti-IL6 R; and 3) Targeted synthetic DMARDs such as Janus kinase inhibitors [13]. Of all these medications, methotrexate continues to be the most widely used treatment for RA because of its immunosuppressive and anti-inflammatory effects as well as low cost. However, methotrexate is not an ideal therapeutic agent due to its toxicity. Methotrexate has adverse effects on the neuronal, gastrointestinal, reproductive, respiratory, urinary, integumentary, cardiovascular and immune systems [14]. Therefore, there is a need to develop better affordable therapeutic agents for RA.

Quercetin (Que) is a natural flavonoid found in vegetables, fruits, grains and nuts [15, 16]. This flavonoid does not cause toxicity or have secondary effects [17-21]. Plants containing quercetin such as Bridelia ferruginea, Betula pendula and Poligonum hydropiper L. have been used in traditional medicine for arthritic pain [22-24]. Que has been described to have several effects including antioxidant, anti-proliferative, antiinflammatory, antihistamine, antiviral, anti-carcinogenic, psychostimulatory, immunosuppressive and protective properties. [16]. Studies in vitro, ex vivo and in vivo indicate that quercetin disturbs different pathways of inflammation. For example quercetin a) inhibits DC and T cell activation, maturation and migration; b) reduces the production of nitric oxide (NO), rheumatoid factor (RF), C-reactive protein (CRP), cytokines, chemokines and prostaglandins (examples include TNF- $\alpha$, IL-1 $\beta$, IL-1 $\alpha$, INF $\gamma$, MCP-1, IL-6, IL-10, IL-12 p70, MIP-1a, MIP-1b, RANTES and PGE-2); c) 
reduces the expressions of costimulatory molecules such as CD40, CD80, and CD86; d) prevents angiogenesis; e) inhibits transcription factors such as NF- $\kappa \beta$; f) reduces enzymes like MMPs, COX, hyaluronidases and collagenases; g) promotes apoptosis of pathogenic cells, and h) inhibits oxidative metabolism [21, 25-33].

In summary, despite methotrexate being an effective drug for RA, it is not specific for the disease and puts at risk the life of patients. Alternative therapies are required to treat RA. In the present study, anti-inflammatory and joint protective properties of quercetin were studied using C57BL/6 collagen-induced arthritis mouse model.

\section{Materials and Methods}

\subsection{Reagents}

Chicken Type II collagen, Complete Freund's Adjuvant (CFA), Incomplete Freund's Adjuvant (IFA), methotrexate and quercetin were purchased from Sigma-Aldrich (St Louis, MO, USA). Phosphate Buffer Saline (PBS) was acquired from Thermo Fisher (Newstead, QLD, Australia). The cytokine kits were purchased from BD Biosciences (North Ryde, NSW, Australia).

\subsection{Induction and evaluation of collagen-induced arthritis}

Mice were injected sub dermally at day 0 with an emulsion containing chicken type II collagen and CFA. Following the first injection, mice received a booster injection at day 21 with an emulsion of chicken type II collagen and IFA. Every five days, collageninduced arthritis was evaluated by visual scoring of the paws including the adjoining area. In addition, swelling in the ankles was measured with digital calipers. Each paw was scored from 0 to 4 as follows: $0=$ normal, $1=$ erythema and mild swelling, $2=$ erythema and swelling extending to ankle joints and one or two toes, $3=$ erythema and swelling extending to metatarsal joints and more than two toes and $4=$ ankylosing deformity with joint swelling. Scores from the four paws were added to get a cumulative score of 0 to 16 . 


\subsection{Treatment groups}

C57BL/6 mice aged 10-12 weeks were obtained from the Small Animal Breeding Facility of James Cook University. The James Cook University Animal Ethics Committee approved all studies. Mice were allocated into five groups according to the treatment: 1) Negative Control (Control), 2) Positive Control (Arthritis), 3) Arthritis + Mtx, 4) Arthritis+Que and 5) Artritis + Mtx + Que.

The negative control group did not receive any treatment. Arthritis was induced in the following groups: Arthritis, Arthritis+Mtx, Arthritis+Que and Arthritis + Mtx + Que. In addition, each mouse from these groups received daily treatments from day 21 to day 70. The Arthritis group received subcutaneous injections of $100 \mu 1$ of PBS. The Arthritis + Mtx group was treated intraperitoneally (i.p.) with $0.5 \mathrm{mg} / \mathrm{kg}$ of methotrexate. The Arthritis+Que was treated daily by oral gavage with $30 \mathrm{mg} / \mathrm{kg}$ of quercetin. The Arthritis + Mtx + Que group was treated with both methotrexate $(0.5 \mathrm{mg} / \mathrm{kg}$ i.p $)$ and quercetin $(30 \mathrm{mg} / \mathrm{kg}$ oral gavage, daily).

\subsection{Cytokine Assay}

Mice were sacrificed by exsanguination under deep anesthesia $24 \mathrm{~h}$ after final treatment (day 71) and blood was collected by cardiac puncture. Plasma was separated from blood by centrifugation ( $1000 \mathrm{~g}$ for 15 minutes). Plasma was stored at $-80^{\circ} \mathrm{C}$ until it was tested to detect pro- and anti-inflammatory cytokines. Quantitative measurement of cytokines was performed with a Quantibody: Multiplex array (BD Pharmigen). Samples were assayed following the protocol of BD Pharmigen in a CyAn ADP (Beckman Counter) flow cytometer.

\subsection{Radiographic Examination}

From each group a mouse was chosen at random and anesthetized at the end of the experiment (day 70). X-ray examination was carried out with a Multi Soft X-ray film apparatus. 


\subsection{Histopathological Analysis}

Left hind knees were dissected out, cleaned and samples were fixed, decalcified and embedded in paraffin to be sectioned. Sections of $5 \mu \mathrm{m}$ were stained with Hematoxylin and Eosin for a blind histopathological examination. Histopathological scoring (Table 1) was performed by a pathologist blinded to the identity of the sample group.

As histopathological scores differed among the groups, the medians of each group including synovitis, pannus formation, cell infiltration and cartilage and bone damage - were obtained to exemplify the arthritic distribution. Further analysis of the minimum, maximum and total scores of synovitis including pannus formation, cell infiltration, cartilage damage, and bone damage was done using column analysis. The means were rounded to the nearest integer and represented as $(-)$ normal, $(+)$ minimum, $(++)$ moderate, and $(+++)$ maximum damage in the knee.

\subsection{Statistical analysis}

GraphPad Prism Version 7.0a was used for data analysis. Data were analysed by twoway ANOVA with Dunnett's multiple comparisons test; one-way ANOVA with Kruskal-Wallis test and Dunn's multiple comparisons test; column statistics and MannWhitney $U$ test. Results are reported as mean \pm SEM. Mean results from the histopathological analysis were rounded to the nearest integer and illustrated with (- ) and $(+)$ symbols. $P$ values below 0.05 were considered statistically significant.

\section{Results}

\subsection{Weight loss and mortality}

Each mouse was weighed at 5 day intervals to determine whether methotrexate and quercetin had an effect on weight gain (Fig. 1A). The mouse weight was not influenced either by arthritis, arthritis+methotrexate or arthritis+quercetin when compared to the control group. However, when comparing the control group with the Arthritis + Mtx + Que group, the weights in the latter group had significantly decreased 
from day 10 to day 70. Mice also lost weight at 45, 55, 60 and 65 days when the Arthritis group was compared with the Arthritis + Mtx+Que group. While none of the other groups showed mortality, the Arthritis+Mtx+Que group had 16.6\% mortality (Table 2). Thus the combined administration of methotrexate and quercetin caused a significant weight loss and mortality in mice.

\subsection{Paw oedema}

All four paws were visually examined and scored for oedema to determine whether quercetin plays a protective role in collagen-induced arthritis (Fig. 1B and Table 2). The negative control group did not show signs of oedema. All mice with CIA showed signs of oedema in the four paws. Mice with arthritis treated with methotrexate or quercetin showed reduced paw oedema from day 50 to day 70 compared to mice with arthritis but without treatment. Mice also showed reduced paw oedema from day 60 to day 70 when methotrexate and quercetin were administered together. The number of mice with a maximum score in any paw was higher in mice with arthritis and mice with arthritis treated with methotrexate. The number of paws with maximum score was higher in mice with arthritis. The maximum score of paw oedema was seen for 10 to 35 days in mice with arthritis, 5 days in mice with arthritis treated with methotrexate or quercetin and 1 day in mice with arthritis treated with methotrexate + quercetin. The cumulative disease decreased significantly in mice treated only with quercetin, particularly the severity of oedema. It is shown that the beneficial effect of methotrexate and quercetin in oedema was delayed when these drugs were administered concurrently. As stated, the administration of methotrexate + quercetin decreased the severity of oedema but not more than quercetin alone.

\subsection{Ankle thickness}

The width of the ankles was measured every five days to determine the role of quercetin in ankle inflammation (Fig. 1C-D). Thickness of the left and right ankles differed significantly between the control group and the arthritis group from day 35 in the left paw and from day 40 in the right paw to day 70 in both paws. Even though the thickness of the left ankle seemed to be lower in mice treated with quercetin compared to those 
with arthritis, significant differences between these groups was seen only on day 65 . No significant differences were observed when the arthritis group was compared with the Arthritis+Mtx group or the Arthritis+Mtx+Que group. These results indicated that quercetin did not have a significant effect in reducing ankle oedema.

\subsection{Radiological examination of the knee joint}

A representative mouse from each group was chosen at random. A radio-graphic image was taken of the hind limbs at 70 days (Fig. 2), to examine the join damage during CIA. Joints showed narrow joint spaces, diffuse soft tissue swelling, cystic enlargement of bone, and erosions in arthritis mice. There was apparent degeneration of joint structure in the mouse with arthritis and no treatment compared to the negative control. However, bone erosion and degradation were not severe, and the extent of the narrowing of joint space was observed to be relatively small in the treatment groups (Fig. 2).

\subsection{Histopathological changes in mice treated with Que and Mtx}

Histopathological evaluation of knees was performed to examine the degree of arthritic damage (Fig. 3A-F and Table 2 and 3). The control group did not show pathological signs of arthritis as illustrated (Fig. 3A-B and Table 2 and 3). The distribution of the histopathological scores of the groups varied significantly $(p=0.0129$ vs Arthritis group by Kruskal-Wallis). Minimal synovitis including pannus formation, inflammatory cell infiltration, cartilage and bone damage was seen in the Arthritis, Arthritis + Mtx, Arthritis + Que and Arthritis + Mtx + Que groups. Maximal synovitis with pannus formation and inflammatory cell infiltration was seen in the Arthritis, Arthritis + Mtx and Arthritis + Mtx + Que groups but not in mice treated with quercetin. The Arthritis+Que group showed moderate synovitis with moderate pannus formation and inflammatory cell infiltration. Maximal cartilage and bone damage was seen in the Arthritis and Arthritis + Mtx + Que groups. The Arthritis + Mtx and Arthritis + Que groups showed moderate damage to cartilage and bone (Fig D and E). 
The Arthritis group showed maximal synovitis including pannus formation with moderate inflammatory cell infiltration, cartilage and bone damage. The Arthritis + Mtx group showed maximal inflammatory cell infiltration with moderate synovitis, pannus formation, cartilage and bone damage. The Arthritis+Que group showed minimal synovitis, pannus formation, inflammatory cell infiltration, cartilage and bone damage. The Arthritis $+\mathrm{Mtx}+$ Que group showed moderate synovitis, pannus formation, cell infiltration, cartilage damage and bone destruction. Interestingly, only mice treated with quercetin showed minimal damage to joints (Fig 3E and Table 2 and 3) clearly indicating that quercetin protected the knee from synovitis, pannus formation, cartilage and bone damage.

\subsection{Effect of treatment on cytokine levels}

At the conclusion of the study, plasma was analysed to find whether changes in cytokine levels influenced the inflammatory changes observed in mice. There were mimimal circulating levels of TNF- $\alpha$, IL-6, IL-1 $\beta$, IL-17, IL- $1 \alpha$ and MCP-1 in the control group when compared with the arthritis group (Figs. 4A-F). Levels of TNF $\alpha$ and IL-1 $\beta$ (Fig. 4A and C) were significantly reduced in groups of mice treated with methotrexate, quercetin and methotrexate + quercetin. Plasma levels of IL-6 (Fig. 4B) were significantly reduced in mice treated with methotrexate+quercetin. IL-17 (Fig. 4D) was significantly decreased in mice treated with methotrexate or quercetin. Levels of IL-1 $\alpha$ (Fig. 4E) were reduced in mice treated with methotrexate. Plasma MCP-1

(Fig. 4F) was decreased in mice treated with quercetin and methotrexate+quercetin. Decreases in the severity of CIA in all groups of mice was associated with lower circulating levels of TNF- $\alpha$ and IL-1 $\beta$. The lowest indicators of arthritis in mice treated with quercetin were associated with significantly reduced levels of IL-17 and MCP-1.

\section{Discussion}

The present study is the first work in vivo examining the therapeutic role of quercetin in CIA in C57BL/6 mice, and comparing the beneficial effects of methotrexate and methotrexate combined with quercetin in experimental RA. The control group did not show signs of either oedema or inflammation (Fig. 1B-D, 2, 3A-B and Tables 2 and 3). Consistent with the absence of the disease, the control group had the lowest levels of 
TNF- $\alpha$, IL-6 and IL-17 (Figs. 4A-B and AF). Although the control group had higher levels of IL-1 $1 \beta$, IL- $1 \alpha$ and MCP-1 than mice treated with methotrexate, quercetin or methotrexate + quercetin, no statistical significant differences were seen between the groups (Fig. 4C and 4E-F). Our finding are in agreement with previous studies in RA and CIA that methotrexate reduces the severity of oedema and inflammatory damage in the joints (Fig. 1B-D, 2, 3D, and Tables 2 and 3) [34-37]. Although methotrexate decreased circulating levels of TNF- $\alpha$, IL-1 $\beta$, IL-17 and IL-1 $\alpha$ (Fig. 4A and 4C-E), it did not have a superior anti-inflammatory or protective effects on the joint tissue compared to quercetin alone.

The present study also found that the administration of both methotrexate + quercetin had no significant combined effect either on the severity of disease or joint destruction when compared to solo therapeutic agent. However the combination of two agents did result in weight loss and death. While Methotrexate has been reportedly caused weight loss and death in RA in a previous study [38], to our knowledge, there are no reports indicating that quercetin causes death in RA or in animal models of the disease. However, studies in vitro showed that quercetin inhibited the differentiation of preadipocytes, suppressed intracellular accumulation of lipids and participated actively in the breakdown of lipids [39]. Death of the mice could be caused because of the toxicity caused by sustained treatment (day 21 to day 70) of methotrexate. Weight loss may be due to a joint action of toxicity, prevention of adipogenesis and acceleration of lipolysis.

Here it is shown that oral administration of quercetin in CIA in C57BL/6 mice decreased paw oedema but not ankle oedema following 4 weeks of treatment (Fig. 1BD, 2 and Table 2 and3). Reduction of paw oedema by quercetin was seen throughout the experiment from day 50 to day 70 . Quercetin also shortened the duration of the maximum severity of oedema. In previous studies Rockland mice with acute inflammation caused by carrageenan had decreased oedema after intraperitoneal (i.p) administration of quercetin [40]. Wistar rats with adjuvant-carrageenan-induced inflammation (ACII) showed reduced acute and chronic paw oedema following i.p administration of quercetin [40,41]. Wistar rats with CFA-induced arthritis had diminished oedema after oral administration of quercetin alone or quercetin-loaded quantum dots (QDs-QE complex) [26]. Decreased oedema after oral or intra-cutaneous 
(i.c.) administration of quercetin was observed in Lewis rats with adjuvant arthritis (AA). The positive properties of quercetin on reducing oedema was not affected by the mode of delivery of the agent. In contrast to the above findings. Choi E et al. [28] found quercetin had no effect in reducing swelling of DBA/1J mice with CIA. Gardini C, et al. [21] also reported that quercetin did not decrease oedema in Lewis rats with AA, however. The clinical signs of arthritis in Lewis rats were only reported for days 14 and 28. Javadi F et al. [18] found that quercetin decreased stiffness and pain but not swelling in women with RA. It is known from previous findings that the effect of quercetin in arthritic inflammation are dose dependent [26, 31]. Choi E et al. [28] fed DBA/1J mice with a diet containing $0.05 \%$ of quercetin for nine weeks. Gardini C, et al. [21] administered $150 \mathrm{mg} / \mathrm{kg} /$ day to Lewis rats for 28 days. Javadi F et al. [18] supplemented women with $500 \mathrm{mg} /$ day of quercetin for 8 weeks. If the beneficial effects of quercetin on the development of oedema are dose dependent, it is possible that the lack of effect of quercetin in mouse studies is possibly related to dose or mouse strain variation. Conflicting results in rats could be related to i.c. administration of quercetin which caused a relapse of the oedema for two days (days 28 and 36) in rats [31]. Although differences in animal models appeared not to be related to the period of administration, extended duration of clinical trials in humans and a reduction in the frequency of assessing for clinical signs could be factors to be considered in future studies.

In our studies, the reduction of oedema was confirmed in the histopathological analysis of the knees. Firstly, we found that the density of inflammatory cells occupying the synovial compartment was minimal in mice treated with quercetin (Figs. 3E, 3G and Table 2). This is not unexpected as it has been previously demonstrated in one study of arthritic rats that white blood cell numbers decrease after QDs-QE complex treatment [26]. This is due to liposomes containing quercetin obstructing DNA binding in targeted APCs in inflammatory arthritis [42]. C57BL/6 mice immunized with OVA and IFA have also demonstrated that quercetin can inhibit Ag-specific T cell activation [43] and inhibit DC Ag loading and blocked DC response to CCL21 in vitro. Therefore it appears that the low cellularity observed in the synovial compartment in our studies is due to the effect of quercetin on activation, maturation, proliferation and migration of inflammatory cells infiltrating the synovial space. Secondly, the group of mice treated with quercetin was the only group showing minimal hyperplasia with limited pannus 
formation (Figs. 3E, 3G and Table 3). Earlier reports indicated that quercetin inhibited HIG-82 rabbit synoviocyte viability and proliferation as well as angiogenesis after stimulation with dimethylthiazol diphenyltetrazolium bromide salt (MTT) [17]. The antiangiogenic effect of quercetin was also demonstrated in vitro and in vivo experiments using human umbilical vein endothelial cells and chicken chorioallantoic membrane respectively [33]. Additionally, Tan WF et al. [33] reported that quercetin inhibited the proliferation of NIH-3T3 fibroblast cells, while studies in RA patients revealed that quercetin induced apoptosis of fibroblast-like synoviocytes (FLS) by activating p53, reducing cytochrome $c$ release and inhibiting Bcl-2, Bax and IL-1 $\beta$ [25, 44]. Apoptosis induced by quercetin has also been reported in DC [43]. This evidence suggests that quercetin prevents hyperplasia and facilitates apoptosis of cells that cause pathology. As synoviocytes and fibroblasts lose their ability to survive and multiply, the formation of fibroblast-type synovial lesions is interrupted. Pannus formation is also prevented as the antiangiogenic properties of quercetin block the growth of new blood vessels.

Quercetin treatment of mice in this study was associated with minimal inflammatory cell infiltration and minimal hyperplasia due to the flavonoid reducing circulating levels of TNF- $\alpha$, IL-1 $\beta$, IL-17 and MCP-1 (Figs. 4A, 4C, 4D and 4F) [21, 28]. Quercetin treatment has also been shown to reduce plasma levels of IL1 $\beta$ in Lewis rats with AA [21] and plasma levels of TNF- $\alpha$ of women with RA [18]. In vitro studies have reported that TNF- $\alpha$ inhibits the activation of NF-k $\beta$, MCP-1 and IL-8 in human synovial cells [45]. Decreased propagation of fibroblasts reduce the level of IL-1 $\beta$ and this decrease in IL-1 $\beta$ affected the production and proliferation of MMPs, COX-2, PGE2; phosphorylation of ERK-1/2, p38, JNK; and activation of NF-kB [17, 44]. It seems that low levels of cytokines after quercetin treatment is consistent with the low cellularity found in the synovial compartment. Moreover, quercetin is known to affects NF-kB activation which decreased cytokine expression [44].

We found that mice treated with quercetin exhibited the highest protection to cartilage and bone than other groups included in the study (Figs. 3A, 3G and Table 3). Minimal degradation in bone and cartilage could be associated with lower circulating levels of IL-17, as this cytokine activates osteoclasts [46]. It has been demonstrated in in vitro 
studies that quercetin regulates glycosaminoglycans and inhibits the expression of MMP3 in proteoglycans [17, 47]. QDs-QE complex also increased levels of SOD, CAT, GPx and GSH, which inhibited cartilage degradation by ROS [26]. Despite the limited information existing about the role of quercetin in cartilage and bone remodelling, it likely that quercetin can directly protect cartilage. It is possible that minimal damage to cartilage and bone that was observed in this study was related to decreased levels of IL-17.

\section{Conclusions}

Despite the therapeutic risks of methotrexate, it has been the most used treatment for RA. Therefore it is essential to continue searching for new drugs to improve patient outcome. This study demonstrated that quercetin could be a potential new therapy for RA. Quercetin diminished the severity of clinical signs of CIA and protected cartilage and bone from destruction. Protection against CIA was associated with decreased circulating levels of TNF- $\alpha$, IL-1 $\beta$, IL-17 and MCP-1. Interestingly, quercetin was shown to be a better anti-inflammatory, immunosuppressive and protective therapy than methotrexate using the CIA model. However, the beneficial properties of quercetin were not enhanced by methotrexate + quercetin; on the contrary it caused significant weight loss in animals and requires further investigation. Quercetin could have a role in the treatment of RA as it decreases the severity of arthritic inflammation, protects joints from degradation and is non-toxic at a therapeutic concentration.

\section{Conflict of interest}

The authors declare no conflict of interest.

\section{Acknowledgement}

The authors would like to thank Arthritis Australia (The HJ \& GJ McKenzie Grant 2015) and Australian Institute of Tropical Health and Medicine, James Cook University for funding this project. 


\section{References}

[1] I. Ahlstrand, M. Bjork, I. Thyberg, B. Borsbo, T. Falkmer, Pain and daily activities in rheumatoid arthritis, Disability and rehabilitation 34(15) (2012) 1245-53.

[2] K. Yamamoto, Y. Okada, A. Suzuki, Y. Kochi, Genetics of rheumatoid arthritis in Asia--present and future, Nature reviews. Rheumatology 11(6) (2015) 375-9.

[3] H. Midtbo, E. Gerdts, T.K. Kvien, I.C. Olsen, M.T. Lonnebakken, E.S. Davidsen, S. Rollefstad, A.G. Semb, The association of hypertension with asymptomatic cardiovascular organ damage in rheumatoid arthritis, Blood pressure 25(5) (2016) 298-304.

[4] L. Tong, J. Thumboo, Y.K. Tan, T.Y. Wong, S. Albani, The eye: a window of opportunity in rheumatoid arthritis?, Nature reviews. Rheumatology 10(9) (2014) 552-60.

[5] C.A. Kelly, V. Saravanan, M. Nisar, S. Arthanari, F.A. Woodhead, A.N. PriceForbes, J. Dawson, N. Sathi, Y. Ahmad, G. Koduri, A. Young, Rheumatoid arthritisrelated interstitial lung disease: associations, prognostic factors and physiological and radiological characteristics--a large multicentre UK study, Rheumatology (Oxford, England) 53(9) (2014) 1676-82.

[6] E. Pathan, V.R. Joshi, Rheumatoid arthritis and the kidney, The Journal of the Association of Physicians of India 52 (2004) 488-94.

[7] G. Titeca, L. Goudetsidis, A. Laka, D. Jardinet, F. Poot, [Rheumatoid neutrophilic dermatitis], Annales de dermatologie et de venereologie 141(10) (2014) 603-6.

[8] S.M. Naz, D.P. Symmons, Mortality in established rheumatoid arthritis, Best practice \& research. Clinical rheumatology 21(5) (2007) 871-83.

[9] C. Terao, S. Raychaudhuri, P.K. Gregersen, Recent Advances in Defining the Genetic Basis of Rheumatoid Arthritis, Annual review of genomics and human genetics 17 (2016) 273-301.

[10] P. Castro-Santos, R. Diaz-Pena, Genetics of rheumatoid arthritis: a new boost is needed in Latin American populations, Revista brasileira de reumatologia 56(2) (2016) 171-7.

[11] D. Alpizar-Rodriguez, N. Pluchino, G. Canny, C. Gabay, A. Finckh, The role of female hormonal factors in the development of rheumatoid arthritis, Rheumatology (Oxford, England) (2016).

[12] S.F. Jalil, M. Arshad, A. Bhatti, J. Ahmad, F. Akbar, S. Ali, P. John, Rheumatoid arthritis: What have we learned about the causing factors?, Pakistan journal of pharmaceutical sciences 29(2) (2016) 629-45.

[13] J.S. Smolen, D. Aletaha, I.B. McInnes, Rheumatoid arthritis, Lancet (London, England) 388(10055) (2016) 2023-2038. 
[14] V.C. Romao, A. Lima, M. Bernardes, H. Canhao, J.E. Fonseca, Three decades of low-dose methotrexate in rheumatoid arthritis: can we predict toxicity?, Immunologic research 60(2-3) (2014) 289-310.

[15] J.M. Harnly, R.F. Doherty, G.R. Beecher, J.M. Holden, D.B. Haytowitz, S. Bhagwat, S. Gebhardt, Flavonoid content of U.S. fruits, vegetables, and nuts, Journal of agricultural and food chemistry 54(26) (2006) 9966-77.

[16] R. Garcia-Mateos, L. Aguilar-Santelises, M. Soto-Hernandez, R. Nieto-Angel, Flavonoids and antioxidant activity of flowers of Mexican Crataegus spp, Natural product research 27(9) (2013) 834-6.

[17] J.K. Jackson, T. Higo, W.L. Hunter, H.M. Burt, The antioxidants curcumin and quercetin inhibit inflammatory processes associated with arthritis, Inflammation research : official journal of the European Histamine Research Society ... [et al.] 55(4) (2006) 168-75.

[18] F. Javadi, A. Ahmadzadeh, S. Eghtesadi, N. Aryaeian, M. Zabihiyeganeh, A. Rahimi Foroushani, S. Jazayeri, The Effect of Quercetin on Inflammatory Factors and Clinical Symptoms in Women with Rheumatoid Arthritis: A Double-Blind, Randomized Controlled Trial, Journal of the American College of Nutrition (2016) 1-7.

[19] Y.D. Min, C.H. Choi, H. Bark, H.Y. Son, H.H. Park, S. Lee, J.W. Park, E.K. Park, H.I. Shin, S.H. Kim, Quercetin inhibits expression of inflammatory cytokines through attenuation of NF-kappaB and p38 MAPK in HMC-1 human mast cell line, Inflammation research : official journal of the European Histamine Research Society ... [et al.] 56(5) (2007) 210-5.

[20] V. Natarajan, N. Krithica, B. Madhan, P.K. Sehgal, Formulation and evaluation of quercetin polycaprolactone microspheres for the treatment of rheumatoid arthritis, Journal of pharmaceutical sciences 100(1) (2011) 195-205.

[21] C. Gardi, K. Bauerova, B. Stringa, V. Kuncirova, L. Slovak, S. Ponist, F. Drafi, L. Bezakova, I. Tedesco, A. Acquaviva, S. Bilotto, G.L. Russo, Quercetin reduced inflammation and increased antioxidant defense in rat adjuvant arthritis, Archives of biochemistry and biophysics 583 (2015) 150-7.

[22] K. Cimanga, L. Ying, T. De Bruyne, S. Apers, P. Cos, N. Hermans, P. Bakana, L. Tona, K. Kambu, D.T. Kalenda, L. Pieters, D. Vanden Berghe, A.J. Vlietinck, Radical scavenging and xanthine oxidase inhibitory activity of phenolic compounds from Bridelia ferruginea stem bark, The Journal of pharmacy and pharmacology 53(5) (2001) 757-61.

[23] C. Grundemann, C.W. Gruber, A. Hertrampf, M. Zehl, B. Kopp, R. Huber, An aqueous birch leaf extract of Betula pendula inhibits the growth and cell division of inflammatory lymphocytes, Journal of ethnopharmacology 136(3) (2011) 44451.

[24] Y. Yang, T. Yu, H.J. Jang, S.E. Byeon, S.Y. Song, B.H. Lee, M.H. Rhee, T.W. Kim, J. Lee, S. Hong, J.Y. Cho, In vitro and in vivo anti-inflammatory activities of 
Polygonum hydropiper methanol extract, Journal of ethnopharmacology 139(2) (2012) 616-25.

[25] P. Xiao, Y. Hao, X. Zhu, X. Wu, p53 contributes to quercetin-induced apoptosis in human rheumatoid arthritis fibroblast-like synoviocytes, Inflammation 36(2) (2013) 272-8.

[26] R. Jeyadevi, T. Sivasudha, A. Rameshkumar, D.A. Ananth, G.S. Aseervatham, K. Kumaresan, L.D. Kumar, S. Jagadeeswari, R. Renganathan, Enhancement of anti arthritic effect of quercetin using thioglycolic acid-capped cadmium telluride quantum dots as nanocarrier in adjuvant induced arthritic Wistar rats, Colloids and surfaces. B, Biointerfaces 112 (2013) 255-63.

[27] J. Lattig, M. Bohl, P. Fischer, S. Tischer, C. Tietbohl, M. Menschikowski, H.O. Gutzeit, P. Metz, M.T. Pisabarro, Mechanism of inhibition of human secretory phospholipase A2 by flavonoids: rationale for lead design, Journal of computeraided molecular design 21(8) (2007) 473-83.

[28] E.J. Choi, S.C. Bae, R. Yu, J. Youn, M.K. Sung, Dietary vitamin E and quercetin modulate inflammatory responses of collagen-induced arthritis in mice, Journal of medicinal food 12(4) (2009) 770-5.

[29] E.O. Santos, L.M. Kabeya, A.S. Figueiredo-Rinhel, L.F. Marchi, M.F. Andrade, F. Piatesi, A.B. Paoliello-Paschoalato, A.E. Azzolini, Y.M. Lucisano-Valim, Flavonols modulate the effector functions of healthy individuals' immune complexstimulated neutrophils: a therapeutic perspective for rheumatoid arthritis, International immunopharmacology 21(1) (2014) 102-11.

[30] J.H. Lee, G.H. Kim, Evaluation of antioxidant and inhibitory activities for different subclasses flavonoids on enzymes for rheumatoid arthritis, Journal of food science 75(7) (2010) H212-7.

[31] M. Mamani-Matsuda, T. Kauss, A. Al-Kharrat, J. Rambert, F. Fawaz, D. Thiolat, D. Moynet, S. Coves, D. Malvy, M.D. Mossalayi, Therapeutic and preventive properties of quercetin in experimental arthritis correlate with decreased macrophage inflammatory mediators, Biochemical pharmacology 72(10) (2006) 1304-10.

[32] C.N. Tran, S.K. Lundy, D.A. Fox, Synovial biology and T cells in rheumatoid arthritis, Pathophysiology : the official journal of the International Society for Pathophysiology 12(3) (2005) 183-9.

[33] W.F. Tan, L.P. Lin, M.H. Li, Y.X. Zhang, Y.G. Tong, D. Xiao, J. Ding, Quercetin, a dietary-derived flavonoid, possesses antiangiogenic potential, European journal of pharmacology 459(2-3) (2003) 255-62.

[34] M.A. Lopez-Olivo, H.R. Siddhanamatha, B. Shea, P. Tugwell, G.A. Wells, M.E. Suarez-Almazor, Methotrexate for treating rheumatoid arthritis, The Cochrane database of systematic reviews (6) (2014) Cd000957. 
[35] N. Lorenzo, F. Altruda, L. Silengo, M. Del Carmen Dominguez, APL-1, an altered peptide ligand derived from heat-shock protein, alone or combined with methotrexate attenuates murine collagen-induced arthritis, Clinical and experimental medicine (2016).

[36] S.W. Lee, J.H. Kim, M.C. Park, Y.B. Park, W.J. Chae, T. Morio, D.H. Lee, S.H. Yang, S.K. Lee, S.K. Lee, S.K. Lee, Alleviation of rheumatoid arthritis by cell-transducible methotrexate upon transcutaneous delivery, Biomaterials 33(5) (2012) 1563-72.

[37] Y.H. Kim, J.S. Kang, Effect of methotrexate on collagen-induced arthritis assessed by micro-computed tomography and histopathological examination in female rats, Biomolecules \& therapeutics 23(2) (2015) 195-200.

[38] C. Salliot, D. van der Heijde, Long-term safety of methotrexate monotherapy in patients with rheumatoid arthritis: a systematic literature research, Annals of the rheumatic diseases 68(7) (2009) 1100-4.

[39] Y.S. Seo, O.H. Kang, S.B. Kim, S.H. Mun, D.H. Kang, D.W. Yang, J.G. Choi, Y.M. Lee, D.K. Kang, H.S. Lee, D.Y. Kwon, Quercetin prevents adipogenesis by regulation of transcriptional factors and lipases in OP9 cells, International journal of molecular medicine 35(6) (2015) 1779-85.

[40] A.E. Rotelli, T. Guardia, A.O. Juarez, N.E. de la Rocha, L.E. Pelzer, Comparative study of flavonoids in experimental models of inflammation, Pharmacological research 48(6) (2003) 601-6.

[41] T. Guardia, A.E. Rotelli, A.O. Juarez, L.E. Pelzer, Anti-inflammatory properties of plant flavonoids. Effects of rutin, quercetin and hesperidin on adjuvant arthritis in rat, Farmaco (Societa chimica italiana : 1989) 56(9) (2001) 683-7.

[42] C. Capini, M. Jaturanpinyo, H.I. Chang, S. Mutalik, A. McNally, S. Street, R. Steptoe, B. O'Sullivan, N. Davies, R. Thomas, Antigen-specific suppression of inflammatory arthritis using liposomes, Journal of immunology (Baltimore, Md. : 1950) 182(6) (2009) 3556-65.

[43] R.Y. Huang, Y.L. Yu, W.C. Cheng, C.N. OuYang, E. Fu, C.L. Chu, Immunosuppressive effect of quercetin on dendritic cell activation and function, Journal of immunology (Baltimore, Md. : 1950) 184(12) (2010) 6815-21.

[44] M.S. Sung, E.G. Lee, H.S. Jeon, H.J. Chae, S.J. Park, Y.C. Lee, W.H. Yoo, Quercetin inhibits IL-1beta-induced proliferation and production of MMPs, COX-2, and PGE2 by rheumatoid synovial fibroblast, Inflammation 35(4) (2012) 1585-94.

[45] M. Sato, T. Miyazaki, F. Kambe, K. Maeda, H. Seo, Quercetin, a bioflavonoid, inhibits the induction of interleukin 8 and monocyte chemoattractant protein-1 expression by tumor necrosis factor-alpha in cultured human synovial cells, The Journal of rheumatology 24(9) (1997) 1680-4.

[46] Y. Lee, The role of interleukin-17 in bone metabolism and inflammatory skeletal diseases, BMB reports 46(10) (2013) 479-83. 
[47] V. Natarajan, B. Madhan, M.L. Tiku, Intra-Articular Injections of Polyphenols Protect Articular Cartilage from Inflammation-Induced Degradation: Suggesting a Potential Role in Cartilage Therapeutics, PloS one 10(6) (2015) e0127165.

Table 1. Histopathological score

\begin{tabular}{cl}
\hline Score & \multicolumn{1}{c}{ Characteristics } \\
\hline 0 & No synovitis and no pannus formation, no cell infiltration, no damage to the \\
cartilage, no damage to the bone & Mild synovitis with limited pannus formation, low cellular density of \\
1 & infiltrating cells, small isolated lesions in the cartilage and small isolated \\
& lesions in the bone \\
& Moderate synovitis with moderate pannus formation, moderate cellular \\
& density of infiltrating cells, moderate lesions in the cartilage and moderate \\
& lesions in the bone \\
Severe synovitis with severe pannus formation, high cellular density of \\
infiltrating cells, extensive damage to the cartilage and extensive damage to \\
the bone.
\end{tabular}


Table 2. Effect of quercetin and methotrexate in CIA in C57BL/6

Treatment Group

\begin{tabular}{|c|c|c|c|c|c|}
\hline & Control & Arthritis & Arthritis + Mtx & Arthritis+Que & Arthritis + Mtx + Que \\
\hline$n$ & 6 & 6 & 6 & 6 & 6 \\
\hline Incidence (\%) & 0 & 100 & 100 & 100 & 100 \\
\hline Mortality (\%) & 0 & 0 & 0 & 0 & 16.6 \\
\hline Maximum score & & 4 & 4 & 4 & 4 \\
\hline $\begin{array}{l}\text { Mean of cumulative } \\
\text { disease } \pm \text { SEM }\end{array}$ & & $5.6 \pm 0.9$ & $3.1 \pm 0.4$ & $3.0 \pm 0.5^{*}$ & $3.4 \pm 0.5$ \\
\hline Number of paws affected & $0 / 0$ & $24 / 24$ & $24 / 24$ & $24 / 24$ & $24 / 24$ \\
\hline $\begin{array}{l}\text { Number of mice with } \\
\text { maximum score in any } \\
\text { paw }\end{array}$ & 0 & 2 & 2 & 1 & 1 \\
\hline $\begin{array}{l}\text { Number of paws with } \\
\text { maximum arthritis score }\end{array}$ & $0 / 24$ & $3 / 24$ & $2 / 24$ & $2 / 24$ & $2 / 24$ \\
\hline $\begin{array}{l}\text { Number of days with } \\
\text { maximum score }\end{array}$ & 0 & $10-35$ & 5 & 5 & 1 \\
\hline
\end{tabular}

Values are from day 20 when mice started to demonstrate clinical signs of disease (one day before start of treatment), ${ }^{*} p<0.05$ and $* * * p<0.0001$ (Arthritis group versus Mtx-Methotrexate; Que-Quercetin and Mtx + Que groups). Medians of the groups varied significantly ( $p<$ $0.0001)$. 
Table 3. Histopathological examination

\begin{tabular}{ccccc}
\hline Mroup & Synovitis & $\begin{array}{c}\text { Cell } \\
\text { infiltration }\end{array}$ & $\begin{array}{c}\text { Cartilage } \\
\text { damage }\end{array}$ & $\begin{array}{c}\text { Bone } \\
\text { damage }\end{array}$ \\
\hline Control & - & - & - & - \\
Arthritis & +++ & ++ & ++ & ++ \\
Arthritis+Mtx & ++ & +++ & ++ & ++ \\
Arthritis+Que & + & + & + & + \\
Arhtritis+Mtx+Que & ++ & ++ & ++ & ++
\end{tabular}

*Mean scores were rounded to the nearest interger and illustrated as: $-=$ Normal, + Minimum,$++=$ Moderate, $+++=$ Maximum. 


\section{Figure Legends:}

\section{Fig 1. Effect of quercetin and methotrexate on body weight and paw edema}

A) Changes in weight of mice. Mice were weighed every five days from the day of arthritis induction (day 0) to day 70. B) Effect of quercetin and methrotexate on paw oedema. Arthritis was assessed by visual scoring of the paws on the scale of 0 to 4 for each paw. The sum of the scores resulted in the cumulative score. C) Effect of methrotrexate on ankle thickness. Thickness of ankles was measured with digital calipers. Each point represents the mean \pm SEM of six mice. Statistical differences are indicated ${ }^{\#} p<0.05$ versus control group; ${ }^{*} p<0.05$ versus arthritis group (Two-way ANOVA with Dunnett's multiple comparisons test).

Fig 2. Representative X-rays of the effect of quercetin and methotrexate. Damage in the joints after 70 days of arthritis induction is indicated with white arrows.

\section{Fig 3. Effect of quercetin and methotrexate on inflammatory changes in CIA.}

A -B Control group showing the main histological structure of the knee joint. Histopathological (20x) changes of the C) arthritis group and groups with arthritis treated with D) methotrexate, E) quercetin and F) methotrexate + quercetin. Cartilage and bone damage is indicated with yellow arrows, pannus is indicated with white arrows, damage in the meniscus is indicated with blue arrows, and areas with fibrosis are indicated with black arrows. 
Fig 4. Effect of quercetin and methotrexate on plasma cytokine levels in collageninduced arthritis in C57/BL6 mice. A- TNF - $\alpha$; B- IL-6; C- IL-1 $\beta$; D- IL-17; E- IL$1 \alpha$; F- MCP-1; Significance: ${ }^{*} \mathrm{P}<0.05 ; * * \mathrm{P}<0.001$ 
A

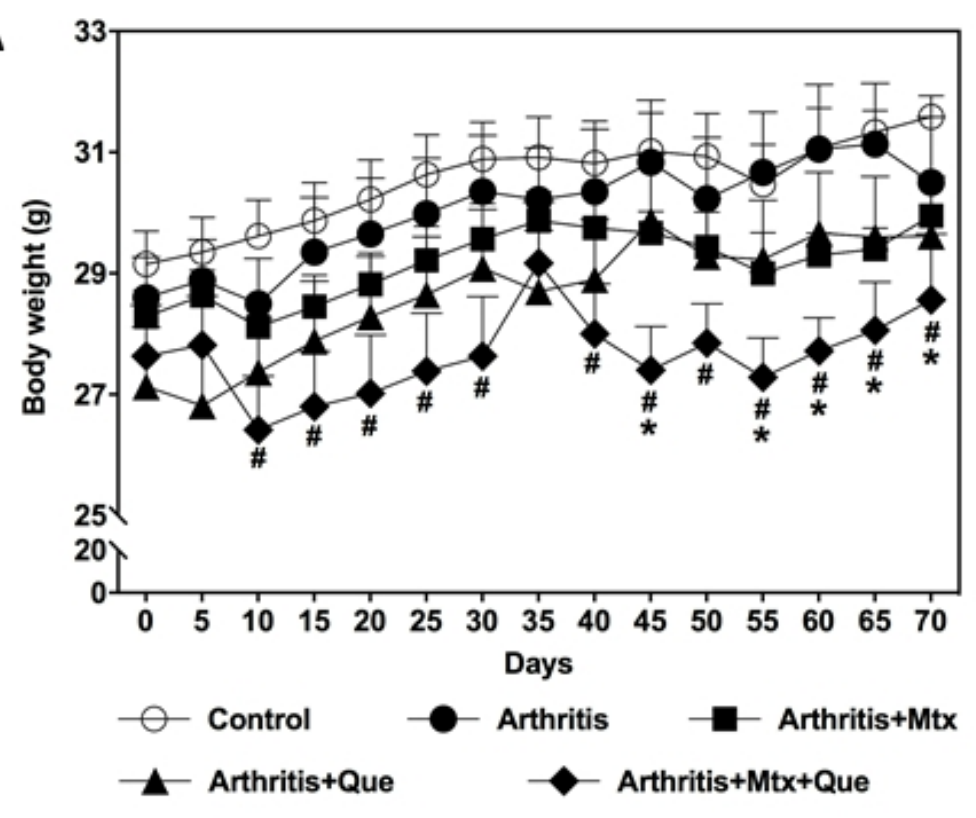

C

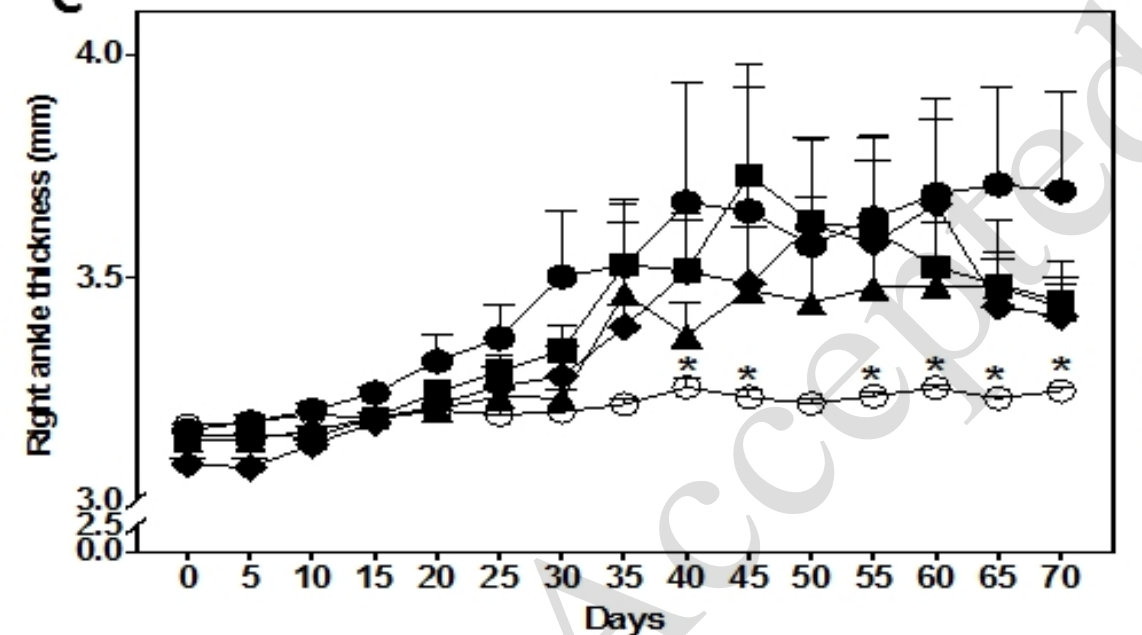

$\rightarrow$ Control $\rightarrow$ Arthritis $\rightarrow$ Arthritis + Mtx

$\multimap$ Arthritis+Que $\leadsto$ Arthritis+Mtx+Que
B

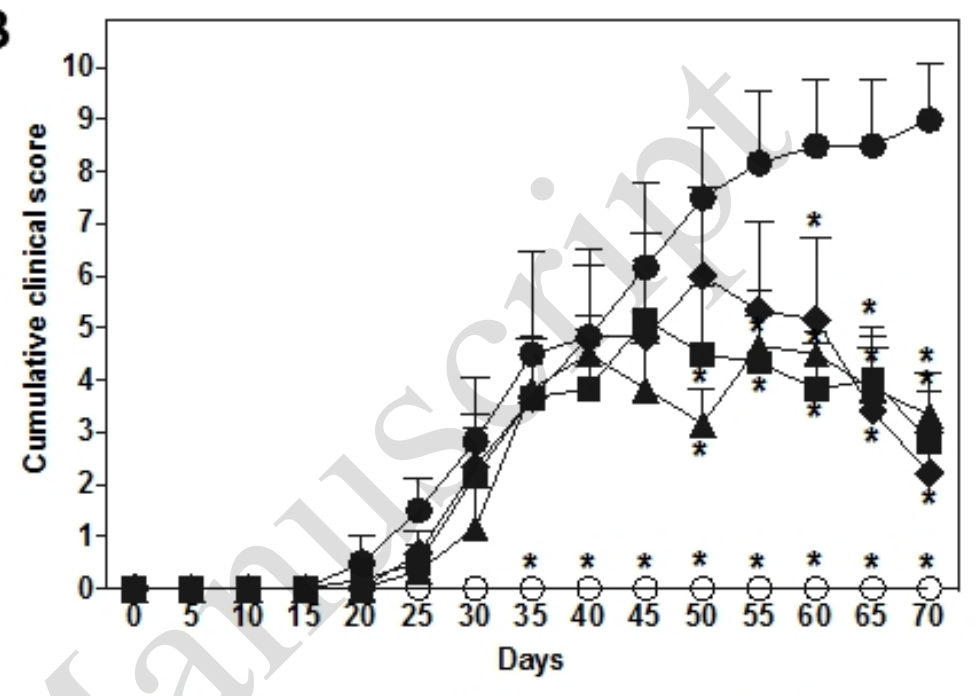

๑ Control

A Arthritis+Que

Arthritis+Mtx+Que

D

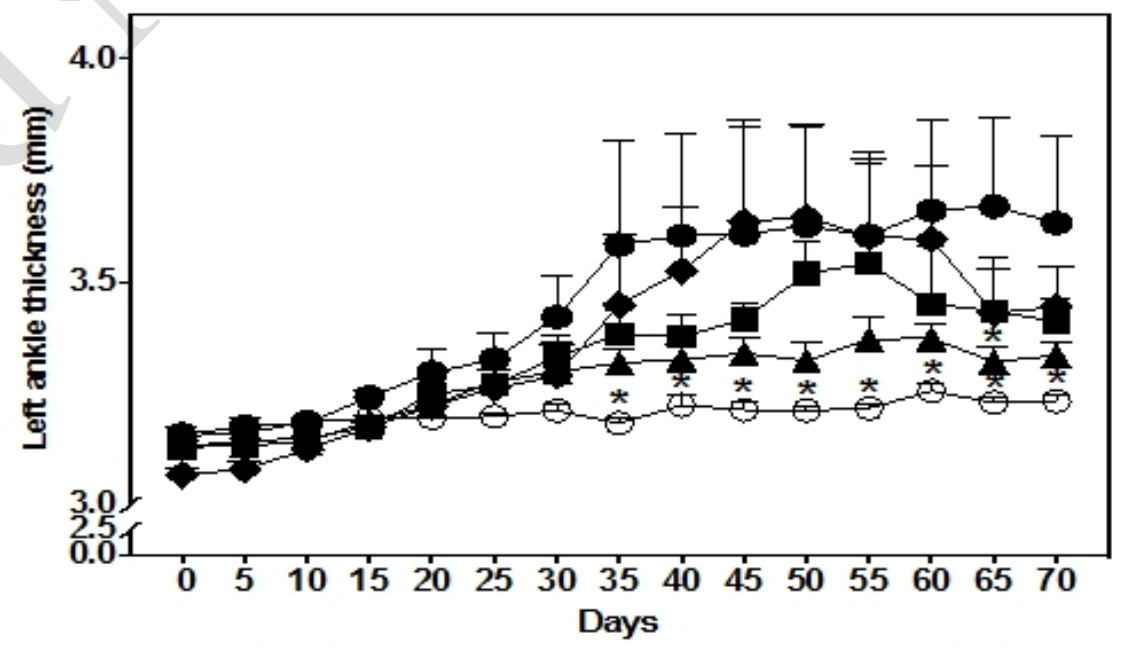

$\rightarrow$ Control $\rightarrow$ Arthritis $\rightarrow$ Arthritis + Mtx

$\longrightarrow$ Arthritis+Que $\longrightarrow$ Arthritis+Mitx+Que 

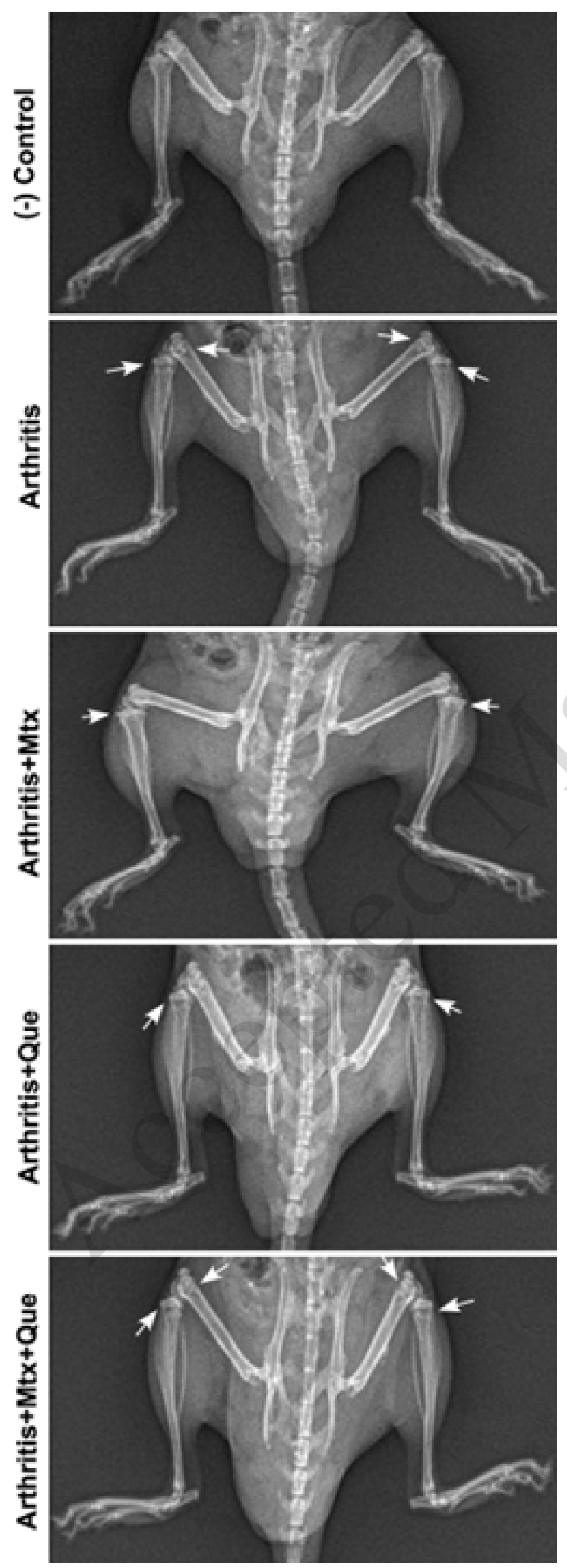

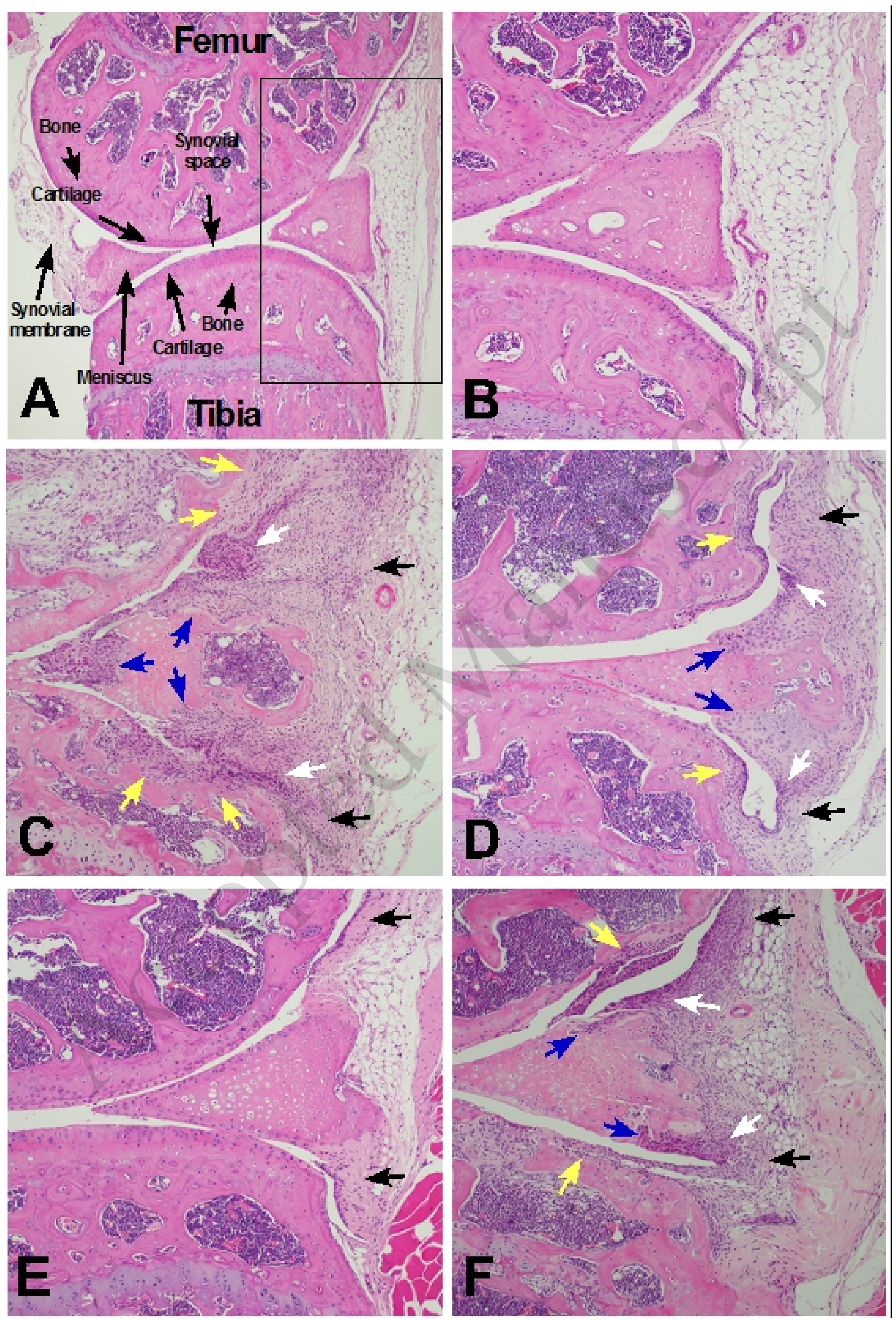

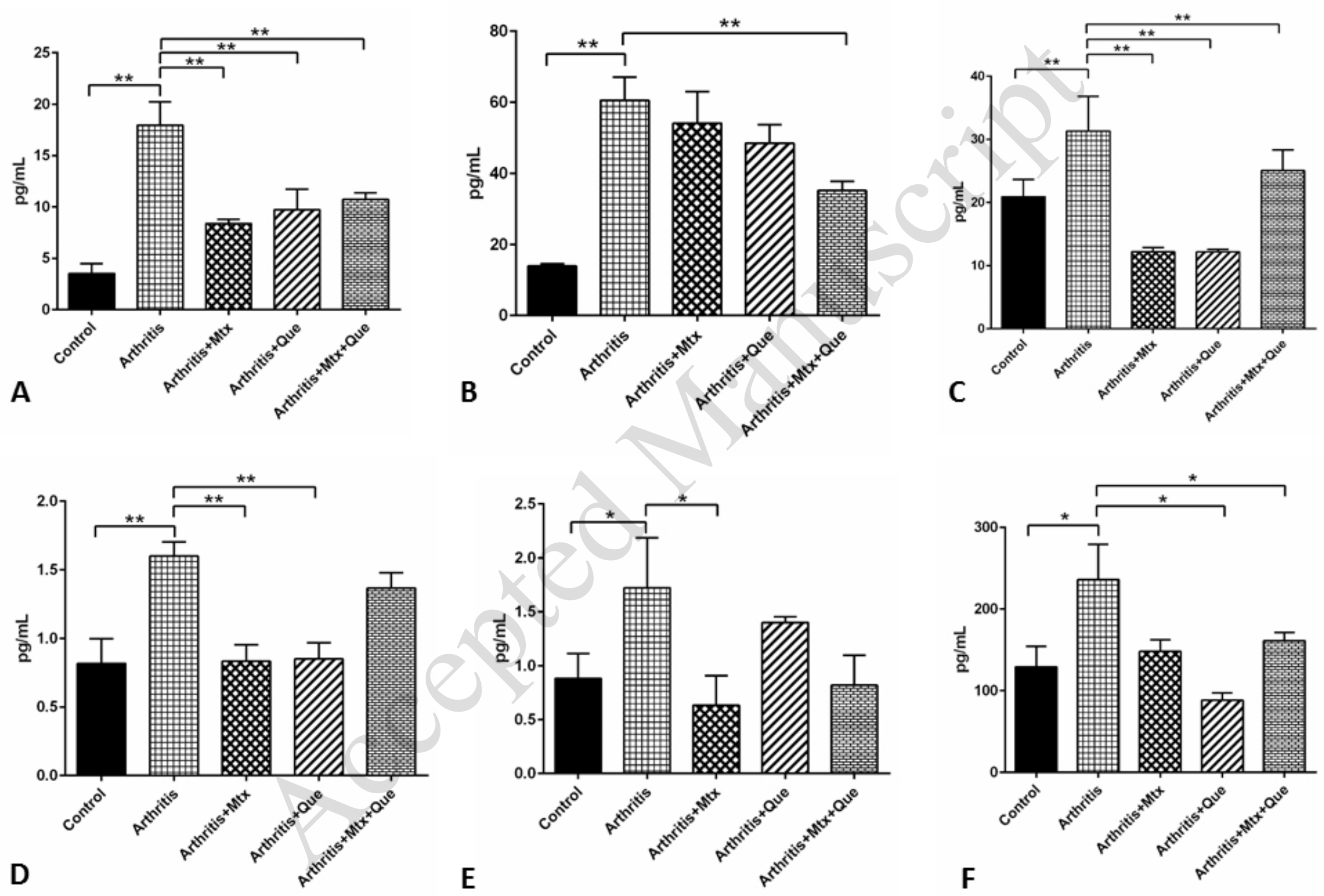